\title{
LA RECHERCHE BIOGRAPHIQUE OU LA CONSTRUCTION PARTAGÉE D'UN SAVOIR DU SINGULIER
}

RÉSUMÉ

\section{ABSTRACT}

\section{CHRISTINE DELORY-MOMBERGER \\ Université Paris 13 Sorbonne Paris Cité}

L'article s'attachera à revisiter les fondements épistémologiques et méthodologiques de la recherche biographique en éducation à partir de deux questions : À quel savoir prétend la recherche biographique? Comment la recherche biographique construit-elle ce savoir ? La première question permettra de réaffirmer la spécificité et la centralité du fait biographique dans les processus d'individuation et de socialisation et d'interroger le champ de connaissance ainsi ouvert à la recherche biographique, en décrivant les opérations et les notions centrales (activité biographique, biographisation, biographicité). La deuxième question conduira à explorer les modes de construction d'un savoir du biographique qui ne peut être, d'une part, qu'un savoir du singulier trouvant sa source en particulier dans les narrations individuelles, et qui ne peut s'édifier, d'autre part, que sur la base d'une recherche collaborative dans laquelle sont engagés ensemble les chercheurs et les personnes sur/avec lesquelles ils "recherchent ». Les éléments de réponse apportés à ce double questionnement seront enfin mobilisés pour tenter d'éclairer la compréhension et l'usage des termes recherche biographique / recherche biographique en éducation pour désigner notre courant de recherche.

Mots-clés: Fait biographique. Récit biographique. Recherche collaborative. Construction partagée du savoir. Education

\section{THE BIOGRAPHY RESEARCH OR THE JOINT}

\section{DEVELOPMENT OF SINGULAR KNOWLEDGE}

This article aims to discuss the epistemic and methodological bases of Biography research in education, throughout two main questions: Which is the knowledge Biography research aims towards? and: How does it develop this knowledge? By describing the operations and key concepts (biographical activity, biographicity, biographization) - the first question will allow us to re-affirm the specificity and the key role of the biography fact within the individuation and socialization 
processes and to question the knowledge field which thus opens up for the Biography research. The second one, will allow us to explore the way biography knowledge is being developed; a knowledge which can - on one hand - only be singular, emerging especially from individual narratives and - on the other - only be developed on the bases of collaborative research, equally involving researchers and the people they work on/ with. The answer premises to theses questions will finally be mobilized in the attempt to clarify the understanding and use of the terms Biography research and Biography research in education for referring to our research field.

Keywords: Biography fact. Biography narrative. Collaborative research. Joint development of knowledge. Education.

\section{LA INVESTIGACIÓN BIOGRÁFICA O LA CONSTRUCCIÓN}

\section{DE UN SABER DEL SINGULAR}

El artículo pretende revisar los fundamentos epistemológicos y metodológicos de la investigación biográfica en Educación a partir de dos preguntas ¿Cuál es el saber que la investigación biográfica busca? ¿Cómo la investigación biográfica construye este saber? La primera cuestión permitirá reafirmar la especificidad y la centralidad del hecho biográfico en los procesos de individuación y de socialización e interrogar el campo de conocimiento abierto para la investigación biográfica, describiendo operaciones y nociones centrales (actividad biográfica, biografización, biograficidad). La segunda cuestión llevará a la exploración de los modos de construcción de un saber del biográfico que no puede ser, de una parte, sino un saber del singular, encontrando su fuente en particular en las narraciones individuales, y que no se puede construir, por el contrario, sino sobre la base de una investigación colaborativa en la cual están comprometidos investigadores y personas sobre/con las cuales ellos “investigan". Los elementos de respuesta traídos a este doble cuestionamiento serán en fin movilizados para tratar de aclarar la comprensión y uso de los términos investigación biográfica/ investigación biográfica en educación, para designar nuestra corriente de investigación.

Palabras clave: Hecho biográfico. Narrativa biográfica. Investigación colaborativa, Construcción compartida de saber. Educación.

\section{RESUMO}

\section{A PESQUISA BIOGRÁFICA OU A CONSTRUÇÃO COMPARTILHADA DE UM SABER DO SINGULAR}

O artigo buscará revisitar os fundamentos epistemológicos e metodológicos da pesquisa biográfica em Educação a partir de duas questões: Qual o saber que a pesquisa biográfica busca? Como a pesquisa 
biográfica constrói este saber? A primeira questão permitirá reafirmar a especificidade e a centralidade do fato biográfico nos processos de individuação e de socialização e interrogar o campo de conhecimento aberto à pesquisa biográfica, descrevendo operações e noções centrais (atividade biográfica, biografização, biograficidade). A segunda questão levará à exploração dos modos de construção de um saber do biográfico que não pode ser, de uma parte, senão que um saber do singular encontrando sua fonte em particular nas narrações individuais, e que não pode se construir, de outra parte, senão na base de uma pesquisa colaborativa na qual estão engajados pesquisadores e pessoas sobre/com as quais eles "pesquisam". Os elementos de resposta trazidos para este duplo questionamento serão enfim mobilizados para tentar esclarecer a compreensão e uso dos termos pesquisa biográfica/pesquisa biográfica em educação para designar nossa corrente de pesquisa.

Palavras-chave: Fato biográfico. Narrativa biográfica. Pesquisa colaborativa. Construção partilhada do saber. Educação

Lorsqu'il s'agit de "science humaine", on ne sait qu'avec les autres.

(Franco Ferrarotti, 2013b)
Une nouvelle fois, ce septième CIPA met au centre de nos débats et de nos échanges les questions d'épistémologie et de méthodologie. C'est que nous n'en avons pas fini d'interroger ce qui fonde le projet et la démarche de la recherche biographique, d'en identifier la spécificité et donc l'autonomie par rapport à d'autres courants des sciences humaines et sociales. Et il ne s'agit pas tant de « se distinguer » que de mieux cerner ce qui est constitutif de notre courant de recherche, de mieux nous représenter à nous-mêmes les objets de connaissance qui sont les nôtres, les notions que nous utilisons, les démarches que nous mettons en œuvre.

Ces questions d'épistémologie et de méthode, je me propose encore une fois d'y revenir, de les "remettre sur le chantier » comme on dit en français, à partir de deux questions dont la simplicité de formulation n'occultera pas le caractère massif: À quel savoir prétend la recherche biographique ? Et: Comment la recherche biographique construit-elle ce savoir?

Disons-le également dès cette introduction: peut-être les éléments de réponse que nous donnerons à ces questions nous amèneront-ils à lever une certaine imprécision dans l'usage et dans l'articulation que nous faisons entre recherche biographique et recherche biographique en éducation.

\section{À quel savoir prétend la recherche biographique?}

S'engager dans la réponse à cette première interrogation, c'est reposer fondamentalement la question du projet épistémologique de la recherche biographique et c'est réaffirmer, 
au cœur de ce projet - pour le dire d'entrée de manière très synthétique -, la spécificité et la centralité du fait biographique. Nous n'aurons pas de mal à nous entendre pour dire que le champ de connaissance de la recherche biographique est celui des processus de constitution individuelle (d'individuation), de construction de soi, de subjectivation, avec l'ensemble des interactions que ces processus engagent avec autrui et avec le monde social. Convenons cependant que la recherche biographique partage ce champ d'investigation avec d'autres courants de recherche comme ceux des sociologies de l'individu ou de la psychologie sociale, en particulier dans leurs versions cliniques. Pour n'en donner qu'un exemple, qui d'entre nous ne pourrait "signer ", au titre même de la recherche biographique, cette définition que donne Florence Giust-Desprairies de la psychologie sociale clinique : « La psychologie sociale clinique est une analyse des processus psychique et sociaux, subjectifs et collectifs, par lesquels le sujet en situation sociale donne sens à son expérience " (GIUST-DESPRAIRIES, 2004, p. 60, c'est nous qui soulignons) ?'À l'intérieur de ce champ de connaissance partagé, il nous faut donc redire que l'entrée spécifique de la recherche biographique, le focus qu'elle se donne et le savoir qu'elle poursuit concernent le biographique en tant que dimension constitutive de la genèse et du devenir socio-individuel.

Comment décliner ce savoir du biographique ? Quels processus singuliers en constituent-ils l'objet ? Quelles notions centrales permettent-elles d'en rendre compte ? Mais aussi quelles finalités en attendons-nous pour

1 D'autant lorsqu'elle poursuit: "Par cette approche, je cherche à situer l'intériorité du sujet dans un contexte, temporalisé et actualisé, de manière à repérer quels enchaînements significatifs se sont formés et continuent à se construire et ce, à partir de quelles sollicitations externes au sujet pour que celui-ci tisse la trame renouvelée de son rapport à luimême, aux autres et au monde. » (GIUST-DESPRAIRIES, 2004, p. 60) la recherche biographique, entre connaissance, éthique et politique ? Ce sont toutes ces questions - qui en soi ne sont pas nouvelles - qu'il nous revient à présent de reprendre de façon plus ciblée dans une volonté d'élucidation et d'approfondissement.

\section{Reconnaître le fait biographique}

La recherche biographique se démarque d'autres courants de recherche en ceci qu'elle introduit la dimension du temps, et plus précisément de la temporalité biographique dans son approche des processus de construction individuelle. L'être humain fait l'expérience de lui-même et du monde dans un temps qu'il rapporte à sa propre existence. La temporalité biographique est une dimension constitutive de l'expérience humaine, à travers laquelle les hommes donnent une forme à ce qu'ils vivent. Cette temporalité biographique a sa grammaire ou sa syntaxe fondée sur la séquence narrative matricielle que représente la mise en intrigue de la vie entre la naissance et la mort. La recherche biographique fait ainsi réflexion de l'inscription de l'agir et du penser humains dans des figures orientées et articulées dans le temps, qui organisent et construisent l'expérience selon la logique d'une raison narrative $^{2}$. Selon cette logique, l'individu humain vit chaque instant de sa vie comme le moment d'une histoire : histoire d'un instant, histoire d'une heure, d'une journée, histoire d'une vie. Quelque chose commence, se déroule, vient à son terme, dans une succession, un chevauchement, un empilement indéfini d'épisodes et de péripéties, d'épreuves et d'expériences. La dimension du biographique doit être ainsi entendue comme une élaboration cumulée et intégrative de l'expérience selon une herméneutique qui fait de la mise en intrigue narra-

2 « Le temps devient temps humain dans la mesure où il est articulé de manière narrative. " (RICOEUR, 1983, p. 17). 
tive son mode d'appréhension et d'intelligibilité de la vie.

Telle est la réalité que nous pourrions dire anthropologique du fait biographique. Les êtres humains n'ont pas un rapport direct, transparent à leur vécu et au déroulement de leur vie ; ce rapport est médiatisé par le langage et par ses formes symboliques. On rappellera la célèbre formule du philosophe allemand Wilhelm Schapp (1992): « Die Geschichte steht für den Mann [L'histoire tient lieu de l'homme] ${ }^{3}$. " L'« écriture de la vie » à laquelle renvoie l'étymologie du mot biographie doit être ici comprise comme une attitude première et spécifique du vécu humain : avant toute traduction ou expression de leur existence dans des formes verbalisées, orales ou écrites, les hommes configurent mentalement leur vie dans la syntaxe du récit. La perception et l'intelligence de leur vécu passent par des médiations qui prêtent une figure narrative aux événements et aux situations de leur existence 4 .

Reconnaissant la dimension constitutive du fait biographique dans le développement humain, le savoir auquel prétend la recherche biographique est d'explorer l'espace et la fonction du biographique dans les processus complémentaires d'individuation et de socialisation, à en interroger les multiples dimensions - anthropologique, sémiotique, cognitive, psychique, sociale - aux fins d'aider à mieux comprendre les liens de production et de construction réciproque des individus et des sociétés. La catégorie du biographique donne

3 « Die Geschichte steht für den Mann » : « L'histoire tient lieu de l'homme " selon la traduction de J. Greisch, ou " l'histoire répond de l'homme » selon celle de P. Ricoeur (1983, p. 114).

4 Ce sont ces médiations que Paul Ricoeur a décrites comme relevant de " la structure pré-narrative de l'expérience temporelle »: " Il n'y a pas d'expérience humaine, observe-t-il, qui ne soit déjà médiatisée par des systèmes symboliques et, parmi eux, par des récits [...] Nous n'avons pas d'accès aux drames temporels de l'existence en dehors des histoires racontées à leur sujet par d'autres ou par nous-mêmes. » (RICOEUR, 1983, p. 113.) en effet accès au travail de genèse socio-individuelle par lequel les individus perlaborent le monde social et historique et ne cessent de le produire en se produisant eux-mêmes.

\section{Décrire l'activité biographique}

C'est ce travail de perlaboration et de genèse que nous devons à présent préciser en resituant et en décrivant l'activité biographique. Il nous faut pour cela revenir aux conditions dans lesquelles ont lieu les expériences que nous vivons (Erlebnis) et à la manière dont nous les construisons comme « expériences » (Erfahrung). Parler de la construction de l'expérience, c'est parler de ce qui est au cœur de l'activité biographique. C'est parler de la manière dont, chacun d'entre nous, nous nous approprions ce que nous vivons, éprouvons, connaissons, dont nous en faisons précisément « de l'expérience ».

Les expériences que nous faisons se déroulent dans les mondes historiques et sociaux auxquels nous appartenons et portent ainsi la marque des époques, des milieux, des environnements dans lesquels nous les vivons. Selon leurs appartenances, leur âge, leur catégories socio-professionnelle, leurs activités de socialité, les individus traversent successivement et quelquefois simultanément un grand nombre d'espaces sociaux et de champs institutionnels : famille, école et institutions de formation, marché du travail, profession et entreprise, institutions sociales et culturelles, associations et réseaux de socialité, etc. Or, ces données sociales " objectives " ne sont pas perçues comme telles dans l'expérience individuelle et singulière qu'ils en font. Si nous prêtons attention à la manière dont les individus vivent et expérimentent la " réalité sociale ", que constatons-nous ? Le mode de présence de l'individu dans le monde social relève d'une expérience dans le temps : l'individu vit l'es- 
pace social comme une succession temporelle de situations et d'événements. Pour l'individu, le monde social se constitue au fur et à mesure de ses expériences comme un ordre sensé d'actions (SCHÜTZ, 1981). Les savoirs sociaux sont organisés dans la conscience individuelle sous forme de scripts d'action et de « plans de vie ». En particulier, les mondes sociaux auxquels il participe sont appréhendés par lui sous la forme des programmes biographiques ou encore des "biographies typiques " dont ils sont porteurs. Chaque espace social (la famille, l'école, l'entreprise, etc.) spécifie ainsi des structures d'action et des profils biographiques qui font partie des savoirs transmis et sont actualisés et éprouvés dans l'expérience quotidienne.

Quels sont les effets de cette « temporalisation biographique » de l'espace social ? Elle entraîne une transformation dans la perception et la construction du monde social, organisé non plus selon des règles abstraites et formelles mais selon le point de vue et la temporalité de celui qui le traverse. Dès lors les réalités sociales n'existent pas pour l'individu comme elles peuvent exister pour le sociologue : elles prennent pour lui l'aspect d'expériences qu'il rapporte à lui-même. Dans la conscience individuelle, les faits sociaux qui déterminent les situations, les interactions, les trajectoires, tout ce qui fait que la vie d'un individu est traversée de part en part par le social, relève de la logique des expériences accumulées et de la forme propre que ces expériences impriment au sentiment de soi-même et de son existence.

L'individu ne peut saisir le social autrement que de manière autoréférentielle, en rapport avec son histoire et ses expériences, dans les formes de son « monde-de-vie», pour reprendre le concept développé par Alfred Schütz (1981), ou encore, selon la belle expression des sociologues allemands Peter Alheit et Bettina Dausien, en construisant « le monde intérieur du monde extérieur »(2000, p. 276). Ce que fait apparaître cette logique de subjectivation et d'appropriation biographique, c'est la dimension socialisatrice de l'activité biographique, le rôle qu'elle exerce dans la manière dont les individus se comprennent eux-mêmes et se structurent dans un rapport de co-élaboration de soi et du monde social. L'activité biographique accomplit ainsi une double et complémentaire opération de subjectivation du monde historique et social et de socialisation de l'expérience individuelle : elle est à la fois et indissociablement ce par quoi les individus se construisent comme êtres singuliers et ce par quoi ils se produisent comme êtres sociaux.

Dans sa dimension socio-historique, l'activité biographique peut être décrite comme un ensemble d'opérations mentales, verbales, comportementales, par lesquelles les individus s' inscrivent subjectivement dans les temporalités historiques et sociales qui leur préexistent et les environnent, en s'appropriant les séquences, les programmes et les standards biographiques formalisés (curriculum scolaire, curriculum professionnel, mais aussi scripts d'action et scénarios) des mondes sociaux auxquels ils participent. Elle apparaît en conséquence comme un processus essentiel de constitution de l'individu en société. La catégorie du biographique pourrait être défini comme une catégorie de l'expérience qui permet à l'individu, dans les conditions de son inscription socio-historique, d'intégrer, de structurer, d'interpréter les situations et les événements de son vécu. Selon les époques et les formes sociétales, les manifestations de l'activité biographique et l'intensité du travail qui lui correspond varient en fonction du recours différencié que font les sociétés à la réflexivité individuelle et à ce champ privilégié de réflexivité que constitue la construction biographique. 
Analyser le processus de

\section{biographisation}

La recherche biographique analyse les processus conjoints d'individuation et de socialisation qui sont constitutifs de la construction et du développement socio-individuel comme des formes d'appropriation et de configuration biographique. C'est pour désigner ce processus d'appropriation/configuration par lequel l'individu produit, pour lui comme pour les autres, les manifestations, le sens et la forme de son existence que la recherche biographique a recours au néologisme de biographisation. Nous ne cessons de rapporter à nousmêmes les situations auxquelles nous sommes mêlés et de faire de ces situations notre expérience ; chaque moment, chaque espace que nous vivons, nous en faisons le moment et le lieu d'une histoire singulière qui est notre histoire : nous ne cessons de fait de nous biographier, c'est-à-dire d'inscrire notre expérience dans des schémas temporels orientés qui organisent mentalement nos gestes, nos comportements, nos actions selon une logique de configuration narrative.

Nous appellerons biographisation l'ensemble des opérations et des comportements par lesquels les individus travaillent à se donner une forme propre dans laquelle ils se reconnaissent eux-mêmes et se font reconnaître par les autres. Cette prise de forme a une dimension réflexive et autoréférentielle dans le sens où elle consiste à rapporter les situations, les événements au foyer d'un soi-même. Dans ce sens, la biographisation apparaît comme une herméneutique pratique, un cadre de structuration et de signification de l'expérience s'exerçant de façon constante dans la relation de l'homme avec son vécu et avec son environnement social et historique.

Quelles sont les formes que prend cette métabolisation de l'expérience ? Si la parole de soi, sous la forme en particulier du récit de la vie, oral ou écrit, est une modalité privilégiée de ces opérations de biographisation - celle qui correspond le plus visiblement à ce processus d'écriture de soi et en constitue la forme la plus élaborée -, il faut lui ajouter bien d'autres formes mentales et comportementales, à commencer par l'apparence physique que nous donnons à voir de nousmêmes (tenue du corps, vêtements, parures), nos manières d'être publiques et privées, nos manières de parler, nos façons $d^{\prime}$ ' " habiter ", nos modes de socialité et de relation, etc. Toutes ces manifestations de soi, implicites ou explicites, conscientes ou inconscientes, participent du sentiment unitaire et intégré que nous avons de nous-même (ipséité) en relation avec la mêmeté de notre existence à travers le temps, et contribuent à forger ce que Ricoeur appelle notre " identité narrative $~^{5}$, soit cette image que nous construisons de nous-même par la médiation narrative, incorporée dans un style, une façon d'être, un mode d'apparition.

Ces opérations de biographisation s'inscrivent dans un processus incessant et cumulatif de structuration et d'interprétation de l'expérience, dont on peut trouver un modèle de description chez les phénoménologues allemands Alfred Schütz et Thomas Luckmann (1979-1984). Selon ce modèle, les individus constituent au fil des situations et des événements qu'ils vivent (erleben) une " réserve de connaissances disponibles » qu'ils utilisent comme système d'interprétation de leurs expériences passées et présentes et qui détermine également la façon dont ils anticipent et construisent les expériences à venir. C'est

5 « La personne, comprise comme personnage de son récit, n'est pas une entité distincte de ses "expériences". Bien au contraire : elle partage le régime de l'identité dynamique propre à l'histoire racontée. Le récit construit l'identité du personnage qu'on appeler son identité narrative, en construisant celle de l'histoire racontée. C'est l'identité de l'histoire qui fait l'identité du personnage. » (RICOEUR, 1990, p. 175) 
cette dimension de "processus continu » de l'expérience entre passé, présent et futur qu'avait relevée et décrite John Dewey (1938) et qui lui avait fait dire que « l'expérience est la vie même ».

Ces « savoirs de l'expérience » ou " ressources biographiques » sont stockés dans la réserve de connaissances disponibles sous la forme de structures d'action généralisées qui formalisent selon une logique biographique les expériences antérieures et pré-figurent les expériences à venir (Alheit, Hoerning, 1989). C'est donc à partir de ces savoirs typisés et de la structure de connaissance qu'ils composent que nous sommes capables de catégoriser et d'intégrer (ou non) ce que nous percevons et ce qui nous arrive, d'accueillir et de reconnaître l'expérience comme « familière », " identique », " analogue », " nouvelle », " étrangère », etc. Le sociologue allemand Peter Alheit (2009, p. 79) a développé la notion de biographicité pour rendre compte de cette capacité d'intégrer de nouvelles expériences à celles que nous avons déjà faites. Nous partageons avec d'autres - et quelquefois avec un grand nombre d'autres - des situations que nous pourrions extérieurement définir comme semblables ou identiques. Mais pour chacun de nous, chaque situation et chaque expérience est singulière, chacun de nous a sa manière particulière de la vivre, de lui donner sa forme et sa signification. Cette singularité ne peut être comprise qu'à travers la logique interne, biographique, de nos expériences antécédentes et la manière dont elles configurent notre appréhension du présent et de l'avenir.

La biographicité est ainsi le code personnel selon lequel nous " lisons » et «parlons » les expériences nouvelles, dont nous nous les « approprions » (aneignen). Toutes les expériences ne présentent pas la même biographicité. Certaines sont facilement intégrées et entrent sans résistance dans notre capital expérientiel ou biographique, parce qu'elles reproduisent des expériences antérieures et que nous pouvons les re-connaître. D'autres expériences demandent un travail d'ajustement, d'interprétation, parce qu'elles ne correspondent pas exactement aux schémas de construction que les expériences passées ont permis de nous approprier. Enfin, dans le quotidien de l'existence, un grand nombre des situations que nous vivons ne sollicitent pas notre conscience active, parce qu'elles correspondent aux scripts répétitifs des cadres sociaux et culturels. Mais, quel que soit le degré de conscience ou d'automaticité auquel répond le processus de biographisation de l'expérience, celui-ci est cependant toujours présent, assurant l'intégration de l'expérience qui advient dans la temporalité et l'historialité propre de l'existence singulière.

Tel est donc le champ de savoir de la recherche biographique articulé autour du fait biographique considéré en lui-même et exploré à travers l'activité biographique et les processus de biographisation. Un tel champ peut être appréhendé sous bien des angles : il peut appeler un regard anthropologique, susciter des approches socio-historiques, être abordé sous l'angle de la littérature et des écritures de soi, nourrir des études linguistiques et discursives; il peut aussi - et c'est le point de vue auquel nous nous tenons essentiellement ici - contribuer à éclairer les dynamiques multi-référentielles de la constitution individuelle dans leur dimension tout ensemble psychique et sociale, physique et symbolique, politique et éducative.

\section{Comment la recherche}

\section{biographique construit-elle son} savoir?

\section{Une « science " du singulier?}

Si tel est le savoir spécifique auquel tend la recherche biographique, comment y at- 
teint-elle, en quoi la constitution de ce savoir requiert-elle des modes d'investigation particuliers ? Le chercheur en recherche biographique ne peut accéder à un " savoir du biographique " que par les entrées que lui donnent les sujets dans les procès de biographisation auxquels ils se livrent. Ceux-ci, nous l'avons dit, peuvent prendre une pluralité de manifestations - mentales, comportementales, gestuelles - mais la médiation privilégiée pour accéder aux modalités singulières selon lesquelles le sujet biographie ses expériences est sans conteste l'activité langagière, la parole que le sujet tient sur lui-même. Parmi toutes les formes du discours (descriptif, explicatif, argumentatif, évaluatif, etc.), une place particulière est reconnue au discours narratif, dans la mesure où le narratif, par ses caractéristiques spécifiques, est la forme de discours qui entretient la relation la plus directe avec la dimension temporelle de l'expérience et de l'agir humain.

Nous avons exposé ailleurs (Delory-Momberger, 2014) le problème épistémologique et méthodologique que pose le recours au récit, dans la difficulté à cerner ce qui s'y joue entre "l'acte de vivre ", " l'acte de raconter » et le texte qui est le produit de l'activité narrative. Dans et par le récit, le sujet accomplit un travail de configuration et d'interprétation - de mise en forme et en sens - de l'expérience vécue. La mise en intrigue narrative a donc une dimension performative ${ }^{6}$ : elle agit, elle produit de l'action, et l'action qu'elle produit s'exerce sur le texte en tant que forme mais elle s'exerce aussi sur l'agir humain dont il est question dans le texte. Les deux dimensions de mise en forme textuelle et de configuration de l'action sont consubstantielles. Le récit n'est donc pas seulement le produit d'un « acte de

6 Est dit performatif un énoncé qui effectue, qui « réalise » l'acte qu'il signifie : par exemple, dire « Je promets » ou « Je jure » ou « Je te baptise », c'est effectuer l'acte de la promesse, du serment ou du baptême. raconter ", il a aussi un pouvoir d'effectuation sur ce qu'il raconte. C'est ce qui fonde l'intérêt premier de la recherche biographique pour le récit, puisque celui-ci, par les opérations de configuration et de métabolisation qu'il met en œuvre, est un puissant " acteur » de biographisation.

Nous rappellerons également que si la question méthodologique est inhérente à la recherche qualitative en général et aux études empiriques auxquelles elle donne lieu (Paillé \& Mucchielli, 2008 ; Olivier de Sardan, 2008), elle est au cœur de la recherche biographique, dans la mesure où celle-ci se fixe pour objet la genèse individuelle du social dans les processus de biographisation. Prise entre la singularité en quelque sorte définitionnelle de son objet et la nécessité d'une formalisation scientifique, la recherche biographique doit se tenir à une position - épistémologique et méthodologique - qui lui permette, non seulement de concilier ces deux exigences, mais de répondre empiriquement à la question qu'elle pose théoriquement, à savoir la fabrication « du monde intérieur du monde extérieur », la métabolisation et l'appropriation par l'individu - et par un individu forcément singulier des environnements de toutes sortes qui sont les siens.

Dans cette entreprise, la recherche biographique, loin d'opposer l'individu - la subjectivité individuelle - et le social comme deux entités séparées qui auraient à s'affronter, s'attache au contraire à tenir ensemble les deux termes d'une relation d'institution réciproque. Le biographique n'est pas seulement un espace de médiation et d'articulation entre l'individuel et le social : il est simultanément le lieu d'une institution de l'individu et d'un accomplissement du social, le champ de leur production réciproque. Se fixant pour objet les modes de constitution de l'individu en tant qu'être social singulier, la recherche 
biographique se doit ainsi de com-prendre, autrement dit de prendre ensemble ce qui constitue précisément l'interface qu'elle se propose d'analyser et de comprendre : à la fois l'agentivité individuelle et la structure sociale, à la fois l'expérience singulière et les " cadres de l'expérience », à la fois la " société vécue » et la " société constituée ». Le devenir biographique est toujours le produit d'une interaction entre l'action des individus et le déterminisme des structures, et la manière dont les personnes rendent compte par le récit (et se rendent compte à elles-mêmes) des voies et processus selon lesquels elles se sont constituées ne peut manquer de recouper les structures synchroniques et diachroniques qui modèlent les parcours individuels. Cependant, en tenant ensemble les deux pôles de la relation individu-société, ce que cherche à saisir la recherche biographique, c'est bien la configuration singulière de faits et de situations, de croyances et de représentations, de valeurs et d'affects selon laquelle les sujets mettent en intrigue leur existence, ce sont bien les constructions de forme et de sens qu'ils donnent à leurs expériences.

Dès lors, lorsque le chercheur (le chercheur en recherche biographique) met en place un espace dans lequel des récits vont pouvoir être tenus et entendus est-il dans une tout autre situation et dans une tout autre disposition que le sociologue (ou l'historien, le démographe, le géographe de l'humain, etc.) lorsque celui-ci " recueille des récits ": pour le sociologue, les récits sont des matériaux qui vont lui permettre de dégager et de constituer des données y compris qualitatives sur les représentations, les croyances, les pratiques, les valeurs des catégories de populations qu'il étudie. Ces éléments composent l'arrière-fond social et culturel qui informe et traverse les existences et les expériences individuelles, et à ce titre ils ne sont pas étrangers au chercheur en re- cherche biographique. Mais l'« intérêt de recherche » de ce dernier est ailleurs : il est dans ce que fait le récit, il le porte à tenter de comprendre comment le récit à la fois produit et donne à voir la construction singulière qu'un individu fait d'une existence et d'une expérience elles aussi singulières qui intègrent et s'approprient ces éléments collectifs.

\section{La construction partagée d'un savoir de l'humain}

Or cette compréhension ne peut être le fruit que d'une construction partagée. Le savoir du singulier auquel tend la recherche biographique ne peut s'édifier que dans une démarche de recherche impliquée dans laquelle sont engagés ensemble les chercheurs et les personnes sur/avec lesquelles ils enquêtent. Franco Ferrarotti, interrogé dans un récent entretien sur la postérité de la conception qu'il défendait de la " méthode biographique » dans son livre fondateur Histoire et histoires de vie ([1983]2013a), répond :

La méthode biographique [...] amène le chercheur à reconnaître qu'il ne sait pas, qu'il ne peut commencer à savoir qu'avec les autres avec les gens -, qu'avec le savoir des gens, et en particulier avec le savoir que ses interlocuteurs - ou ses « interacteurs » - construisent avec lui dans des prises de paroles, dans des conversations, dans des récits. [...] Et cela demande d'être dans un contact immédiat avec les personnes ou les groupes sur lesquels on enquête, de développer des rapports d'interaction, une relation interpersonnelle complexe et réciproque, dans laquelle le chercheur est lui-même un "recherché". Et de fait ces personnes participent tout autant que vous à la recherche et c'est à ce titre que l'on peut parler de co-construction ou de construction partagée. Dans cette interaction de recherche, il n'y pas un enquêteur ou un observateur qui connaît et un enquêté ou un observé qui est connu. Comme nous le savons, l'observateur est complètement impliqué dans le champ de son objet qui en est lui-même mo- 
difié. La connaissance n'a pas l'autre pour objet mais l' interaction réciproque entre observateur et observé, c'est une connaissance à deux qui se construit dans une interaction, dans une intersubjectivité. » (FERRAROTTI, 2013b, p. 23)

Les êtres humains ne sont pas des " données » que l'on pourrait connaître depuis le regard extérieur et objectif auquel a longtemps prétendu le savoir positiviste des sciences de la nature ; ils sont des " processus en devenir », des êtres ancrés dans l'espace et dans le temps, des " sujets mobiles et incertains » (FERRAROTTI, 2011). Toute science de l'humain, toute prétention ou ambition à édifier un «savoir de l'homme » (et non pas un "savoir sur l'homme ») doit dès lors intégrer dans ses fondements épistémologiques comme dans ses principes méthodologiques cette dimension processuelle, cet ancrage contextuel, ce caractère d'imprédictibilité. Ce savoir de l'homme ne peut donc relever que d'une science de l'incertitude (FERRAROTTI, 2005) ancrée dans une écologie des savoirs7. Et comme le chercheur " de science humaine » participe par sa propre humanité à l'ensemble de ces traits, qu'il est inévitablement et sans doute indissociablement " pris » et " compris » dans son " sujet d'étude », que son désir, sa volonté, sa quête de savoir rencontrent le désir, la volonté, la quête d'autrui, sa recherche ne peut être elle-même qu'un processus de médiation, d'intercession, l'exploration d'un entre, l'émotion et le mouvement d'un écart8, qui supposent, engagent, appellent la présence de l'autre, reconnaissent sa part créative dans le moment d'un « vivre commun » et dans l'espace d'un " parler ensemble ». La recherche en sciences humaines, si elle est fidèle à elle-même et cohérente avec

7 Sur ce thème, on lira avec beaucoup d'intérêt les liens que fait Elsa Lechner (2012) entre les positions de Franco Ferrarotti et le paradigme de "l'écologie des savoirs » développé par le sociologue portugais Boaventura de Sousa Santos.

8 En cette matière, la pensée du sinologue François Jullien (2012) ne peut manquer d'être convoquée. ce qu'elle prétend, a ceci de singulier qu'elle ne peut être qu'une quête partagée, qu'elle ne peut s'édifier que sur la collaboration - sur le travail mené ensemble - de personnes ou de groupes qui sont simultanément enquêteurs et enquêtés et qui vivent, agissent, parlent, construisent en commun ce qui constitue entre eux l'œuvre de connaissance. Il s'agit dès lors, pour reprendre les mots de l'anthropologue Éric Chauvier rejoignant pleinement le propos de Franco Ferrarotti, de " concevoir le processus de l'enquête comme objet même de l'enquête » (2011, p. 56). Invitant à une véritable " conversion du regard ", cette conception d'une science humaine comme expérience d'un vécu et d'une parole partagés doit conduire à sortir définitivement d'un paradigme scientifique où les positions d'" objet » et de "sujet » sont établies de principe et de manière invariable, où l'universel s'oppose au singulier comme le général au particulier.

\section{Un espace de recherche clinique}

Par son objet et sa méthode, nous l'avons dit, la recherche biographique engage des démarche dans lesquelles enquêteurs et enquêtés sont dans un processus commun de recherche et de connaissance qui, s'il ne vise pas le même savoir pour les uns et pour les autres, présuppose leur interdépendance et leur collaboration. Comme l'écrit avec beaucoup de pertinence Anne Dizerbo dans un travail non publié :

" La recherche biographique est par essence collaborative puisqu'elle implique un travail du sujet avec lequel le chercheur entre en relation, en même temps qu'un travail du chercheur. Mais ce travail n'est pas de même nature pour les uns ou les autres. Pour faire simple : les sujets travaillent à mettre en sens leur expérience ; les chercheurs travaillent à mettre en sens le travail que font les sujets en mettant en sens leur expérience. » 
À ce titre, l'espace de recherche de la recherche biographique ne peut être qu'un espace de relation ou comme le dit encore Ferrarotti, de "dynamique relationnelle ", dont témoigne la centralité accordée à la parole des acteurs et à «l' interaction sociale » que constitue en particulier la situation d'entretien :

Restituons à l'entretien biographique toute son épaisseur d'interaction sociale. Les récits biographiques dont nous nous servons ne sont pas des monologues face à un observateur réduit au rôle de support humain d'un magnétophone. Chaque entretien biographique est une interaction sociale complexe, un système de rôles, d'attentes, d'injonctions, de normes et de valeurs implicites, souvent aussi de sanctions. [...] Liaisons entre observateur et observé dans une relation réciproque, connaissance scientifique exigeant l'herméneutique d'une telle interaction, récit biographique perçu comme action sociale, l'entretien biographique nous semble être un exemple parfait du pôle clinique des sciences humaines. » (FERRAROTTI, 2013a, p. 54 et 56)

Ce « pôle clinique » que constitue aux yeux de Franco Ferrarotti l'entretien biographique, nous le reprendrions bien volontiers à notre compte, si l'on entend par clinique l'espace d'une relation où se déploient un soin et une écoute de l'autre ici attentifs (attentionnés) à ce qui se joue pour la personne d'une quête des formes et du sens de ses expériences. La relation qui s'établit dans l'entretien de recherche biographique ouvre en effet un double espace heuristique : celui du chercheur et de l'objet de sa recherche, mais aussi celui de l'« enquêté » appelé, à travers le récit auquel il est invité, à mettre en œuvre un travail de recherche et de mise en forme de son expérience. Cette double entreprise de recherche, celle de l'enquêté en position d'enquêteur de lui-même, celle du

9 Le livre de Christophe Niewiadomski, Recherche biographique et clinique narrative (2012), offre le cadre théorique pour penser cette dimension clinique et en développe d'éclairantes illustrations. chercheur dont l'objet est de comprendre le travail de l'enquêté sur lui-même, invitent à interroger les positions de l'enquêteur et de l'enquêté, du questionneur et du questionné. Qui est le véritable questionneur dans « l'entretien de recherche biographique » ? Celui qui se parle et se raconte ou celui qui écoute et qui reçoit ? Celui qui est à l'épreuve de son récit et, à travers lui, de ses formes d'existence, ou celui qui recueille les preuves de ce questionnement ? Et qui est le véritable questionné ? Celui qui, à travers son récit, met en œuvre l'herméneutique pratique de son existence ou celui qui cherche à entendre et à comprendre ce travail de l'interprétation ? Ne sont-ils pas, l'un et l'autre, questionneur et questionné, enquêteur et enquêté ? N'ont-ils pas leur part, l'un et l'autre, à la construction d'une œuvre de connaissance qui, pour n'avoir pas le même sens pour l'un et pour l'autre, n'est pas autrement possible que dans le partage qu'ils en font?

Cette dimension relationnelle et clinique qui est au cœur de notre espace de recherche interroge assurément nos démarches et nos finalités scientifiques mais aussi notre responsabilité éthique et politique. Et sans doute fautil revenir un instant sur ce à quoi nous engage, nous chercheurs, d'avoir affaire à ce « type de matériau » très particulier que constitue la parole des acteurs. Les entretiens narratifs, les récits biographiques contribuent à constituer une connaissance en situation, une compréhension de l'intérieur des vécus humains. Ils font accéder à la manière dont des acteurs singuliers vivent, pensent et agissent leurs vies dans les contextes qui sont les leurs; contre les discours dominants et les savoirs hégémoniques, ils font advenir "le point de vue du sujet » et les types de savoir qu'il élabore au fil de son expérience : un savoir qui se construit toujours dans une localité historiquement, socialement, et sémiotiquement située et qui 
renvoie - pour reprendre un terme de Donna Haraway - à la perspective « encorporée » des lieux, des conditions et des points de vue à la fois collectifs et singuliers d'où il est parlé.

Que faire alors de ces "savoirs encorporés » du vécu et de l'expérience ? Comment les traiter, comment les faire signifier ? Comment les articuler à d'autres formes de savoirs ? Ce qui est peut-être une façon de dire, plus radicalement : que faire des " sujets ", que faire de la parole des sujets dans l'étude et le traitement des questions qui les concernent ? Je pense que la réponse - très simple intellectuellement et très complexe si on veut la traduire dans les faits - est dans la question : il faut faire avec les gens, il faut faire avec les sujets. Pour les chercheurs, cela signifie mettre en place des dispositifs de recherche participative, de partage et d'hybridation des savoirs, de co-construction de la connaissance ; pour les responsables institutionnels et politiques, cela signifie instituer des espaces et des pratiques de gouvernance démocratique qui ne se suffisent plus de la seule délégation représentative mais qui intègrent la parole, le point de vue et la capacité de décision des acteurs concernés. S'il y a là un horizon utopique, c'est cet horizon qui donne à mon sens à la recherche biographique, outre sa portée scientifique, sa pleine dimension de clinique politique.

\section{Conclusion}

Au terme de cette revisitation de nos objets et de nos démarches de savoir, je souhaiterais conclure en revenant sur ce qu'elle me semble pouvoir éclairer dans l'usage que nous faisons des termes recherche biographique et recherche biographique en éducation pour désigner notre courant de recherche.

À côté du domaine institué de la formation et de l'éducation, c'est tout ce qui fait « l'établissement humain », c'est, pour reprendre les mots de Lucien Sève, " l'ensemble des processus biographiques à travers lesquels l'individu de l'espèce humaine devient psychiquement sociétaire du genre humain » (SEVE, 2008 , p. 105) qui constitue l'espace dévolu à la recherche biographique, dès lors que celle-ci se donne pour tâche d'en appréhender l'expérience subjective. Traduit dans le langage éthico-politique du sujet et de la Cité, ce long processus d'individuation/socialisation, qui est la marque du développement humain et qui traverse tous les espaces de l'action et de l'expérience humaine, n'est pas autre chose que le mouvement d'éducation par lequel l'être humain sort de sa minorité ${ }^{10}$. Dans ce sens, la recherche biographique conçoit "l'éducation » comme une des dimensions constitutives du fait et du devenir humains : dans l'espace social et dans le temps de l'existence, il s'agit toujours de comprendre comment se forme et se construit l'être social singulier.

Ce sont par ailleurs les travaux menés au titre de la recherche biographique sur les relations entre processus de construction biographique et processus d'apprentissage et de formation qui montrent les liens étroits entre éducation et individuation, éducation et socialisation, et qui amènent à redéfinir la notion d'éducation, conçue comme un processus large qui recouvre toutes les formes de l'expérience vécue et acquise. De la dimension globale du développement individuel dans l'espace social et la vie personnelle aux épisodes spécifiques vécus dans les institutions d'enseignement et de formation, des formes expérientielles de formation et d'apprentissage rencontrées dans l'activité professionnelle et dans la vie sociale au rôle des environnements et des médiations socioculturels, ce sont toutes les expériences,

10 Sur ce thème éminemment kantien, voir le beau livre de Cynthia Fleury, Les irremplaçables : " S' individuer, devenir Sujet, nécessite de sortir de l'état de minorité dans lequel on se trouve, naturellement et symboliquement. » $(2015$, p. 16) 
tous les espaces, tous les types de formation et d'apprentissage, formels, non-formels et informels se déployant tout au long de la vie, qui composent le champ d'un apprentissage biographique concernant la globalité de la personne.

De par la définition même de son projet, la recherche biographique se trouve presque toujours du côté de formes de recherche qui, menées ou co-construites avec les acteurs, poursuivent selon les cas et à des degrés divers, des objectifs individuels et collectifs de formation, de valorisation des ressources et des potentialités, de production et de partage des savoirs, d'émancipation, de pouvoir d'agir, de transformation sociale et politique.

Cette préoccupation de "l'éducation humaine » donne à la recherche biographique une responsabilité particulière, qui n'est pas seulement d'ordre scientifique mais aussi éthique et politique. Si la " parole de soi ", sous tous ses registres et dans toutes ses variétés, constitue le matériau privilégié d'un savoir du biographique, elle est aussi le vecteur par lequel les êtres humains accèdent à un savoir et à un pouvoir d'eux-mêmes qui les

\section{Références}

ALHEIT, P. « Diskurspolitik » : Lebenslanges Lernen als postmodernes Machtspiel ? [« Politique du discours »: l'apprentissage tout au long de la vie comme enjeu de pouvoir dans la postmodernité ?]. In P. Alheit, H. Felden (Hrsg.). Lebenslanges Lernen und erziehungs-wissenschaftliche Biographieforschung [Apprentissage tout au long de la vie et recherche biographique en éducation]. Wiesbaden : VS Verlag für sozialwissenschaften, 2009, p. 77-88.

ALHEIT P., HOERNING E. (Hrsg). Biographisches Wissen. Beiträge zu einer Theorie lebensgeschichtlicher Erfahrung [Savoirs biographiques. Contributions à une théorie de l'expérience totu au long de la vie]. Frankfurt/New York : Campus, 1989.

ALHEIT, P., DAUSIEN, B. Die biographische Konstruk- mettent en capacité de se développer et d'agir en tant que " sujets " au milieu des autres et au sein de la cité. À ce point où le vocabulaire prétendument neutre de la science cède devant le langage de l'éthique et du politique, la recherche biographique ne peut manquer d'être interpellée par la très inégale répartition sociale et géographique des ressources directement liées à la capacité des personnes d'élaborer et de faire entendre sur elles-mêmes un langage recevable, d'accéder aux moyens d'expression et d'affirmation qui les feront socialement et politiquement reconnaître. De telles questions renvoient la recherche biographique à ce qui constitue la dimension éthique de sa démarche, à savoir la préoccupation d'éclairer les conditions sous lesquelles la parole de soi peut constituer pour le sujet un vecteur d'appropriation de son histoire et de son projet et contribuer ainsi à une perspective " émancipatrice » des personnes et des groupes humains. Pour toutes ces raisons, on l'aura compris, la recherche biographique telle que nous l'entendons ne peut être théorisée et pratiquée que comme recherche biographique en éducation.

tion der Wirklichkeit. Überlegungen zur Biographicität des Sozialen. [La construction biographique de la réalité. Réflexion sur la biographicité du social]. In E. M. Hoerning (dir.), Biographische Sozialisation. [Socialisation biographique]. Stuttgart : Lucius \& Lucius, 2000.

CERTEAU, M. de. L'invention du quotidien 1. Arts de faire. Paris : Gallimard Folio, 1990.

CHAUVIER, É. Anthropologie de l'ordinaire. Une conversion du regard. Paris : Anacharsis Éditions, 2011.

DELORY-MOMBERGER, C. Histoire de vie et recherche biographique en éducation. Paris : Anthropos, 2005. DELORY-MOMBERGER, C. (2007). Biographisches 
Lernen [Apprentissage biographique]. In M. Göhlich, C. Wulf, J. Zirfas (dir.). Pädagogische Theorien des Lernens [Théories pédagogiques de l'apprendre]. München : Beck Verlag, 2007, p. 142-152.

Delory-Momberger, C. La recherche biographique. Projet épistémologique et perspectives méthodologiques. In. : De la recherche biographique. Fondements, méthodes, pratiques. Paris : Téraèdre, 2014, p. 73-94.

DEWEY, J. Experience and Education. New-York: The MacMillan Company, 1938

FERRAROTTI, F. On the science of uncertainty. The biographical method in social research. Oxford/ New York: Lexington Books, 2005.

FERRAROTTI, F (2011). Las historias de vida como método. Acta sociológica, 56, 95-119.

FERRAROTTI, F. Histoire et histoires de vie. Préface de Georges Balandier, introduction de Antonella de Vicenti et Gaston Pineau. Paris : Téraèdre, 2013a [1983].

FERRAROTTI, F. Partager les savoirs, socialiser les pouvoirs. Entretien avec Christine Delory-Momberger. Le sujet dans la Cité. Revue internationale de recherche biographique, 4, 19-27, 2013b.

GIUST-DESPRAIRIES, F. Le désir de penser. Construction d'un savoir clinique. Paris : Téraèdre, 2004.

JULLIEN, F. L'écart et l'entre. Leçon inaugurale de la chaire sur l'altérité. Paris : Éditions Galilée, 2012.

LECHNER, E. La recherche biographique aujourd'hui.
De la science de l' incertitude au savoir ancré. Le sujet dans la Cité. Revue internationale de recherche biographique, 2, 214-226, 2012.

NIEWIADOMSKI, C. Recherche biographique et clinique narrative. Entendre et écouter le Sujet contemporain. Toulouse : Érès, 2012.

OLIVIER DE SARDAN, J.-P. La rigueur du qualitatif. Les contraintes empiriques de l'interprétation socio-anthropologique. Louvain-la-Neuve : Academia-Bruylant, 2008.

PAILLE, P. \& MUCCHIELLI, A. L'analyse qualitative en sciences humaines et sociales. Paris : Armand Colin, 2008.

RICOEUR, P. Temps et récit I. Paris : Seuil, 1983.

RICOEUR, P. Soi-même comme un autre. Paris : Seuil, 1990.

SCHAPP, W. Empêtrés dans des histoires. L'être de l'homme et de la chose, Traduction de J. Greisch. Paris : Éditions du Cerf, 1992.

SCHÜTZ, A. Der sinnhafte Aufbau der sozialen Welt [La construction sensée du monde social]. Frankfurt/M. : Suhrkamp, 1981.

SCHÜTZ A., LUCKMANN T. Strukturen der Lebenswelt [Structures du monde-de-vie]. 2 vol. Frankfurt/M. : Suhrkamp, 1979 et 1984.

SEVE, L. Penser avec Marx aujourd'hui. Tome 2 : « L'homme » ? Paris : La Dispute, 2008.

Reçu: 05/01 / 2016

Accepté: 04/02/2016

Christone Delory-Momberger est Professeur des universités Sciences de l'éducation Paris 13 Sorbonne Paris Cité. Centre de recherche interuniversitaire EXPERICE. Présidente Collège International de Recherche Biographique em Education et Directrice scientifique de Le sujet dans la Cité - Revue internationale de recherche biographique. E-mail : christine.delory@lesujetdanslacite.com 